Acknowledgements: The research leading to these results has received support from the Innovative Medicines Initiative Joint Undertaking under grant agreement $n^{\circ} 115565$, resources of which are composed of financial contribution from the European Union's Seventh Framework Programme (FP7/2007-2013) and EFPIA companies' in kind contribution.

Disclosure of Interest: None declared

DOI: 10.1136/annrheumdis-2018-eular.6544

FRIDAY, 15 JUNE 2018

Scleroderma, myositis and related syndromes

\section{FRI0425 ASSOCIATION BETWEEN TISSUE OXYGEN DELIVERY AND DIGITAL ULCERS IN SYSTEMIC SCLEROSIS}

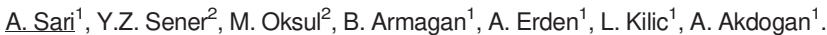
${ }^{1}$ Rheumatology; ${ }^{2}$ Cardiology, Hacettepe University Faculty of Medicine, Ankara, Turkey

Background: Digital ulcers (DUs) are common manifestations of vascular disease in systemic sclerosis (Ssc) patients.

Objectives: To investigate the association between the amount of oxygen deliv ered to periferal tissues and the development of DUs in SSc patients.

Methods: One hundred and eleven SSc patients (103 female) were recruited consecutively to the study. Thirty age and sex matched controls were also enrolled. Transthoracic echocardiography (TTE) was performed for each patient to estimate cardiac output (CO). Arterial oxygen saturation ( $\mathrm{SaO} 2)$ with pulse oximeter in room temperature and serum haemoglobin concentration $(\mathrm{Hb})$ were measured at the time of TTE. The amount of oxygen delivered to tissues (D02) in 1 min was estimated using the equation $\mathrm{D} 02=\mathrm{CO}^{*}\left(\mathrm{SaO}^{*} 1.39^{*} \mathrm{Hb}\right)$ and adjusted for body weight. Demographic, clinical and laboratory data were retrieved from hospital records. DUs were described as ulcerations on the skin distal to proksimal interphalangeal joints (PIF) of the digits. Patients were divided into 2 groups according to presence of DUs within 1 year of study enrollment (Group1=with DU, Group2=without DU). Chi-square and Mann-Whitney U tests were used for comparing categorical and continuous variables between the groups, respectively. Results: Mean age was 52.6 (11.8) years in SSc patients and $51.3(13.0)$ in controls. Median disease duration was 138.0 (1.8-415.2) months. Diffuse disease was present in $30.6 \%$ of patients. There were no differences in terms of $\mathrm{Hb}$ level, left ventricular ejection fraction (LVEF) and calculated D02 between SSc patients and controls. Mean $\mathrm{CO}$ was higher in healthy controls ( $6.1 \mathrm{vs.} 5.1, \mathrm{p}=0.004)$. Interestingly, mean S02 measurements was lower in control group (96.8 vs. 98.0, $\mathrm{p}=0.005$ ). Comparison of demographics, disease characteristics, tissue oxygen delivery and relevant variables were summarised in table 1 . When patients with active DUs were compared with patients without active DUs; no difference was found in $\mathrm{Hb}, \mathrm{S} 02, \mathrm{CO}$ and $\mathrm{D} 02$ between groups.

Abstract FRI0425 - Table 1. Comparison of demographics, disease characteristics and DO2 parameters between patients with and without DUs

\begin{tabular}{|c|c|c|c|}
\hline & Group 1(n=64) & Group 2 (n=47) & p \\
\hline Age, years(SD) & $52.3(12.0)$ & $49.9(14.4)$ & 0.34 \\
\hline $\begin{array}{l}\begin{array}{l}\text { Disease duration,month } \\
\text { (SD) }\end{array} \\
\end{array}$ & $141.0(96.8)$ & $177.3(97.4)$ & 0.06 \\
\hline Female,n (\$6) & $58(90.6)$ & $44(93.6)$ & 0.56 \\
\hline \begin{tabular}{|l} 
Diffuse disease, $n(\$ 6)$ \\
\end{tabular} & $11(17.2)$ & $23(48.9)$ & $<0.01$ \\
\hline ILD,n\% & $32(50.0)$ & $35(74.5)$ & 0.01 \\
\hline PAH,n(\$6) & $3(4.7)$ & $1(2.1)$ & 0.63 \\
\hline Ever smoked, n(36) & $16(25.0)$ & $13(27.6)$ & 0.82 \\
\hline$H T, n(\%)$ & $15(23.4)$ & $6(12.7)$ & 0.22 \\
\hline$D M, n(\% 6)$ & $12(18.7)$ & $3(6.3)$ & 0.09 \\
\hline Cardiac involvement, $n(\$ 6)$ & $1(1.5)$ & $1(2.1)$ & 1.0 \\
\hline $\mathrm{Hb}, \mathrm{g} / \mathrm{dL}$ & $12.8(1.4)$ & $12.2(1.4)$ & 0.04 \\
\hline $\mathrm{SO2,96(SD)}$ & $97.8(2.5)$ & $98.3(1.1)$ & 0.13 \\
\hline $\mathrm{CO}, \mathrm{ml} / \mathrm{min}(\mathrm{SD})$ & $5.1(1.5)$ & $5.1(1.6)$ & 0.91 \\
\hline $\mathrm{DO2}, \mathrm{ml} / \mathrm{min} / \mathrm{kg}$ (SD) & $13.6(4.5)$ & $14.3(4.6)$ & 0.49 \\
\hline
\end{tabular}

Conclusions: Although the haemoglobin concentration was higher in the non ulcer group, there was no difference in the amount of delivered oxygen between patients with and without DUs. These findings imply that vasculopathy and/or peripheral artery disease plays a major role in the development of ulcers.

Disclosure of Interest: None declared

DOI: 10.1136/annrheumdis-2018-eular.4902

\section{FRI0426 RESPONSE OF THE REACTIVE SPECIES INTERACTOME TO HYPOTHERMIA INDUCED RAYNAUD'S ATTACK IN PRIMARY RAYNAUD AND SYSTEMIC SCLEROSIS}

A. Eman Abdulle ${ }^{1}$, A.M. van Roon ${ }^{1}$, A.J. Smit ${ }^{1}$, A. Pasch ${ }^{2}$, M. van Meurs ${ }^{3}$ H. Bootsma ${ }^{4}$, B.O. Fernandez ${ }^{5}$, M. Feelisch ${ }^{5}$, H. van Goor ${ }^{6}$, D.J. Mulder ${ }^{1}{ }^{1}$ Dept of Internal medicine, div Vascular medicine, University of Groningen - University Medical Centre Groningen., Groningen, Netherlands; ${ }^{2}$ Dept. of Clinical Research, University of Bern and Calciscon, Bern, Switzerland; ${ }^{3}$ Dept. of Critical Care; ${ }^{4}$ Dept. of Rheumatology and Clinical Immunology, University of Groningen - University Medical Centre Groningen., Groningen, Netherlands; ${ }^{5}$ Dept. of Clinical and Experimental Sciences, University of Southampton - Southampton, Southampton, UK; ${ }^{6}$ Dept. of Pathology and Medical Biology, Section Pathology, University of Groningen - University Medical Centre Groningen., Groningen, Netherlands

Background: Although the exact etiology of systemic sclerosis (SSc) remains unknown, a current hypothesis is that reactive species (including reactive oxygen species (ROS), reactive nitrogen species (RNS and reactive sulfur species (RSS)) play an important role in the pathogenesis. In addition, the chemical interactions between these molecules, also known as the reactive species interactome, may lead to the formation of more harmful free radicals, thus leading to more damage. We have previously shown a rapid free thiol increase in SSc patients during a cooling and recovery experiment, however the implications of RNS and RSS in this process remain uncharacterized.

Objectives: The aim of the current study was to explore nitric oxide and sulfate response, as a measure of the unified reactive species interactome, during hypothermia induced Raynaud's attack in systemic sclerosis (SSc), primary Raynaud's phenomenon (PRP) and healthy controls (HC)

Methods: Healthy controls $(n=10)$, PRP patients $(n=10)$ and SSc patients $(n=10)$, all aged $\geq 18$ years, were included. All SSc patients fulfilled the ACR/EULAR 2013 criteria. Fingertip photoelectric plethysmography was performed during a standardised cooling and recovery experiment. Patient characteristics were obtained and blood was drawn at four predetermined time points (T0, T1, T2 and T3). In addition to the previously mentioned free thiols, the following markers were also measured: nitros $(\mathrm{yl})$ ated species, nitrite, nitrate, sulfate and the vascular leakage related molecules angiopoietin-1 and angiopoietin-2).

Results: Our results revealed a significantly longer median duration of hypoperfusion and longer recovery time in SSc patients compared to PRP patients and HC This coincided with stable levels of angiopoietin-1/angiopoietin-2 throughout the experiment. In regards to the thiol modification related molecules, (nitrate, nitrite and RXNO) an increased median level of nitrate (T2: median $29.6 \mu \mathrm{mol}$, range 21.99-41.70 $\mu \mathrm{mol}, \mathrm{p}=0.041$ ) was found in SSc patients. Correspondingly, the same trend was observed for the median plasma concentrations of sulfate ( $p>0.05$ at all time points)

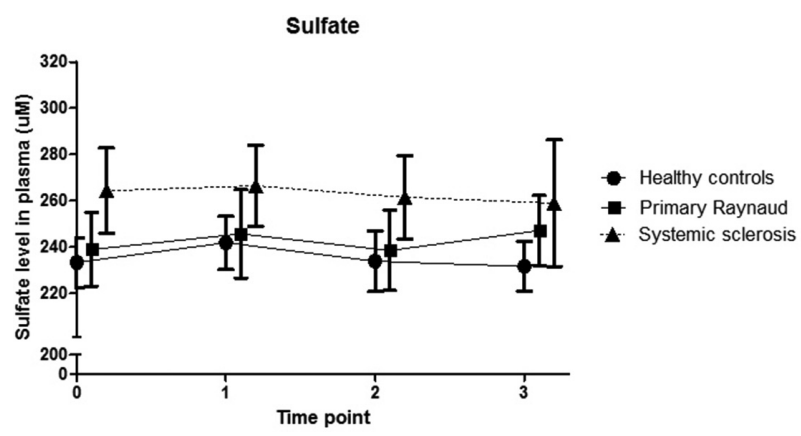

Conclusions: We have previously shown an increase of free thiols in all patients during the reperfusion phase. This finding sheds new light on the possibility that processes related to oxidative thiol modification, are rapidly reversible. The current findings revealed also a trend in increased levels of nitrate and sulfate in SSc patients. Therefore, it is plausible that these markers of the redox system interact with one another through the same pathway, underlining the complexity of the redox interactome and suggesting potential novel approaches for therapeutic intervention.

Disclosure of Interest: None declared

DOI: 10.1136/annrheumdis-2018-eular.7051 